\title{
SIMULATION OUTPUT ANALYSIS BASED ON EXCURSIONS
}

\author{
James M. Calvin \\ Department of Computer Science \\ New Jersey Institute of Technology \\ Newark, NJ 07102-1982, U.S.A.
}

\begin{abstract}
We consider the steady state output analysis problem for a process that satisfies a functional central limit theorem. We construct asymptotically valid confidence intervals for the steady-state mean using normalized excursions of the simulated process. An alternative approach splits the output at the last zero crossing of the normalized path. The methods use a bounded memory of size determined by the user.
\end{abstract}

\section{INTRODUCTION}

A basic simulation problem is to construct asymptotically valid confidence intervals for the steady-state mean of a simulated stochastic process. One of the most commonly used and widely applicable methods is the method of batch means. This method can be applied when the simulated process satisfies a functional central limit theorem (described below). The idea is to break up the suitably normalized and centered simulated path into a fixed number of segments (batches), and to compute a statistic based on the increments over the segments; see Goldsman and Schmeiser (1997). Variations include the method of overlapping batch means (Meketon and Schmeiser 1984).

In this paper we consider methods that break the simulated path into random length segments. In some settings there can be computational advantages, as well as advantages in terms of statistical efficiency. The methods are standardized time series methods, but we center the process by running two (or more) independent simulations in parallel and taking their difference. For background on standardized time series methods, see Schruben (1983) or Glynn and Iglehart (1990).

The methods we describe are partly motivated by the problem of simulation-based optimization. Some optimization algorithms use a large collection of estimates based on different parameter values. It must be possible to halt and resume simulations at different parameter values as those parameters become more or less important for identifying the optimum. Thus it is important for the simulations to use bounded memory (since there may be many of them going on in parallel), and it is not known in advance how long the simulation at a particular parameter value will ultimately run.

In the next section we describe the basic setup and the use of ranked excursions for constructing asymptotically valid confidence intervals. In Section 3 we describe the straight-forward batching method. An alternative approach, based on blocking the output at the last zero crossing, is presented in Section 4. The results of some numerical experiments are presented in Section 5.

\section{RANKED EXCURSIONS}

Let $Y=[Y(t): t \geq 0]$ be a process representing the output of a simulation. If the output is a discrete sequence $\left[Y_{0}, Y_{1}, \ldots\right]$ then set $Y(t)=Y_{\lfloor t\rfloor}$. For each $n \geq 1$, define the scaled process

$$
Y_{n}(t)=\frac{1}{n} \int_{s=0}^{n t} Y(s) d s \quad 0 \leq t \leq 1 .
$$

Let $\Omega_{0}$ be the space of continuous real-valued functions on the unit interval that vanish at 0 . The scaled process $Y_{n}$ has paths that are in $\Omega_{0}$ even if the simulation output $Y$ is not continuous.

Suppose that there is a real number $\mu$ and a positive number $\sigma$ such that if we define a sequence of processes $X_{n}, n \geq 1$, by

$$
X_{n}(t)=\sqrt{n}\left(Y_{n}(t)-\mu t\right), \quad 0 \leq t \leq 1,
$$

then

$$
X_{n} \stackrel{d}{\rightarrow} \sigma B
$$

in $\Omega_{0}$ endowed with the uniform metric as $n \rightarrow \infty$, where $B$ is a standard Brownian motion, and $\stackrel{d}{\rightarrow}$ denotes convergence in distribution. In this case we say that the process satisfies a functional central limit theorem. 
In discrete-event simulation, we are often interested in estimating the parameters $\mu$ and $\sigma$. Suppose that one is interested in constructing an asymptotically valid confidence interval for $\mu$ using only a bounded amount of memory. In particular, the simulated sample path can not be stored. We now outline such a procedure.

Run two independent simulations, say $Y_{1}$ and $Y_{2}$. Set

$$
A_{i}(t)=\int_{s=0}^{t} Y_{i}(s) d s
$$

for $i=1,2$, and define the centered process $A(t)=A_{1}(t)-$ $A_{2}(t)$. Set

$$
X_{n}(t)=\frac{1}{\sqrt{n}} A(n t), \quad 0 \leq t \leq 1 .
$$

Let $\left[\alpha_{i}, \beta_{i}\right], i \geq 1$, denote the intervals of excursion above the minimum for the process $A$. That is,

$$
A\left(\alpha_{i}\right)=A\left(\beta_{i}\right)=\min _{s \leq \alpha_{i}} A(s),
$$

and

$$
A(s)>A\left(\alpha_{i}\right)
$$

for $\alpha_{i}<s<\beta_{i}$. Set

$$
M_{i}=\sup \left\{A(s)-A\left(\alpha_{i}\right): \alpha_{i} \leq s \leq \beta_{i}\right\}
$$

and

$$
l_{i}=\beta_{i}-\alpha_{i} .
$$

We call the process $A$ over the interval $\left[\alpha_{i}, \beta_{i}\right]$ the $i$ th excursion above the minimum. For the $i$ th excursion, consider the pair $v_{i}=\left(l_{i}, M_{i}\left(l_{i}\right)^{-1 / 2}\right)$ (we refer to the second component as the normalized excursion height). Fix a number $m$; the amount of storage will be proportional to $m$. Let $k_{n}$ denote the number of excursions above the minimum that have occurred by simulated time $n$. As the simulation progresses, store the $m$ elements of $\left\{v_{i}: 1 \leq i \leq k_{n}\right\}$ with the largest lengths $l_{i}$; a priority queue keyed on $l_{i}$ could be used for this purpose. Let $H_{i, n}$ be the normalized height of the $i$ th longest excursion above the minimum after simulated time $n$ ( $=0$ if $i>k_{n}$ ). For the following theorem we fix $m \geq 1$ and consider the $m$ longest excursions.

Theorem 1 As $n \rightarrow \infty, H_{i, n} / \sigma$ converges in distribution to the height of a standard Brownian excursion. Furthermore,

$$
\begin{aligned}
& \left(H_{1, n}, H_{2, n}, \ldots, H_{m, n}, X_{n}(1)\right) \\
& \quad \stackrel{d}{\rightarrow} \sigma \cdot\left(H_{1}, H_{2}, \ldots, H_{m}, \sqrt{2} N\right),
\end{aligned}
$$

where $N \sim N(0,1)$ and the $H_{i}$, the maximum of normalized excursions, are independent.

Theorem 1 is proved in Calvin (2004).

Let $B$ be a standard Brownian motion, and suppose that

$$
B(\alpha)=B(\beta)=0,
$$

and

$$
B(s)>0 \quad \forall s \in(\alpha, \beta) .
$$

Then the process

$$
e(t)=\frac{B(\alpha+t(\beta-\alpha))}{\sqrt{\beta-\alpha}}, \quad 0 \leq t \leq 1,
$$

is a standard (positive) Brownian excursion; see Revuz and Yor (1994). Let $M$ denote the maximum of a standard Brownian excursion. Then

$$
P(M \leq x)=\sqrt{2} \pi^{5 / 2} x^{-3} \sum_{n=1}^{\infty} n^{2} \exp \left(-\frac{1}{2} \pi^{2} \frac{n^{2}}{x^{2}}\right) .
$$

The mean of the distribution is $\sqrt{\pi / 2}$.

As a point estimator of $\mu$ we take

$$
\mu_{n}=\frac{1}{2 n}\left(A_{1}(n)+A_{2}(n)\right) .
$$

Then

$$
\sqrt{n}\left(\mu_{n}-\mu\right) \stackrel{d}{\rightarrow} \sqrt{2} \sigma N(0,1) .
$$

Let

$$
V_{n, m}=\frac{1}{m} \sum_{i=1}^{m} H_{i, m},
$$

and let $V_{m}$ denote the average of $m$ independent standard excursion heights. Then

$$
\frac{\sqrt{n / 2}\left(\mu_{n}-\mu\right)}{V_{n, m}} \stackrel{d}{\rightarrow} \frac{N(0,1)}{V_{m}} .
$$

Let $G_{m}$ denote the cumulative distribution function of the limit random variable in (3). For $m=1$ we have

Theorem 2

$$
G_{1}(x)=1-\pi x^{2} \sum_{n=1}^{\infty} n K_{1}(\pi n x),
$$

where $K_{1}$ is the modified Bessel function. The 95th quantile is approximately 1.39739.

It appears difficult to obtain a useful expression for $G_{m}$, $m>1$; simulation can be used to approximate quantiles. 
Thus if we keep only the longest excursion above the minimum, then a $90 \%$ confidence interval for $\mu$ can be constructed as

$$
\left[\mu_{n}-1.39 \cdot H_{1, n} /(2 \sqrt{n}), \mu_{n}+1.39 \cdot H_{1, n} /(2 \sqrt{n})\right] .
$$

\section{BATCHING}

A natural alternative to the method described in the previous section is to use the increments over a fixed grid. Suppose that we store $\left\{X_{n}(i / m): i=0,1,2, \ldots, m\right\}$ for some fixed $m$. Then

$$
\frac{\sqrt{n}\left(\mu_{n}-\mu\right)}{\left(\frac{1}{m} \sum_{i=1}^{m}\left(X_{n}(i / m)-X_{n}((i-1) / m)\right)^{2}\right)^{1 / 2}} \stackrel{d}{\rightarrow} t_{m},
$$

where $t_{m}$ denotes a $t$ distribution with $m$ degrees of freedom. In our setup the limit $t$ distribution has one more degree of freedom than for the usual batch means since we have a Brownian motion limit instead of a Brownian bridge.

As $n$ increases, one could "forget" some of the values to maintain a memory of size $m$. Thus we can construct a method for which the run length would not need to be known in advance, though it would be cumbersome.

\section{BLOCKING ON LAST ZERO CROSSING}

The method described in the previous section uses evaluations of the normalized path concentrated in a relatively small subset of the unit interval (on average less than half, since the portion after the global minimum is not used). In this section we consider an alternative approach that uses evaluations that are more spread out, though the number of "batches" $m$ can not be increased in the version we describe here.

Let $T_{n}$ be the location of the last zero crossing of $X_{n}$, so that $X_{n}\left(T_{n}\right)=0$ and $\left|X_{n}(s)\right|>0$ for $T_{n}<s \leq 1$. Let

$$
R_{n}=\frac{\max _{0 \leq s \leq T_{n}} X_{n}(s)-\min _{0 \leq s \leq T_{n}} X_{n}(s)}{\sqrt{T_{n}}},
$$

and

$$
U_{n}=\frac{\left|X_{n}(1)\right|}{\sqrt{1-T_{n}}} .
$$

Let $R$ denote the range of a standard Brownian bridge. Then $R$ has the same distribution as the random variable $H_{1}$ of Theorem 1 (Vervaat 1979). Let $M_{1}$ denote the value at 1 of a standard Brownian meander (roughly speaking, a Brownian motion conditioned to take positive values over $(0,1])$. Then (Revuz and Yor 1994, p. 468) $M_{1}$ has the same distribution as $\sqrt{2 \gamma}$, where $\gamma$ has an exponential distribution with mean 1 .
Theorem 3 As $n \rightarrow \infty$,

$$
\left(R_{n}, U_{n}\right) \stackrel{d}{\rightarrow} \sigma \cdot\left(H_{1}, M_{1}\right),
$$

where $H_{1}$ and $M_{1}$ are independent, $H_{1}$ is the maximum of a standard Brownian excursion, and $M_{1}$ is the value at time 1 of a standard Brownian meander.

For scaling function we take $\left(R_{n}^{2}+U_{n}^{2} / 2\right)^{1 / 2}$, and so the limit random variable is

$$
\frac{N}{\left(H_{1}^{2}+\gamma\right)^{1 / 2}}
$$

where $N \sim N(0,1)$ and all three random variables are independent. Then for $z>0$,

$$
\begin{gathered}
P\left(\frac{N}{\left(R^{2}+\gamma\right)^{1 / 2}} \leq z\right)= \\
=\frac{1}{\sqrt{1+2 / z^{2}}}+ \\
2 \sum_{k=1}^{\infty}\left(\frac{2 k^{2}+1}{\left(2 k^{2}-1\right)^{2}}\left(1+2 / z^{2}\right)^{-1 / 2}\right. \\
-\frac{4 k^{2}}{z^{2}\left(2 k^{2}-1\right)}\left(1+4 k^{2} / z^{2}\right)^{-3 / 2} \\
\left.\quad-\frac{2 k^{2}+1}{\left(2 k^{2}-1\right)^{2}}\left(1+4 k^{2} / z^{2}\right)^{-1 / 2}\right) .
\end{gathered}
$$

For a derivation, see Calvin (2004).

\section{EXPERIMENTS}

This section describes some numerical experiments that compare the characteristics of the excursion and the batching estimators. The simulated model is the Ehrenfest urn model with state space $\{0,1, \ldots, 8\}$. This is the discrete-time Markov chain with transition probabilities given by: $P_{0,1}=$ $P_{8,7}=1$, and

$$
P_{i, i+1}=\frac{8-i}{8}=1-P_{i, i-1}, \quad 0<i<8 .
$$

We ran 1, 000 independent replications, each replication having a run length of 1,000,000 transitions. In Table 1 we plot the coverage of nominal $90 \%$ confidence intervals, and the average and standard deviation of the confidence interval half-widths.

In Table 2 we plot similar results for the excursionbased estimator. It appears that coverage degrades, while the size and variability of the half widths decrease. 
Table 1: Coverage, Average and Standard Deviation of Half-Widths for Confidence Intervals Based on $m$ Batches

\begin{tabular}{|l|l|l|l|}
\hline$m$ & coverage & avg. hw & std. of hw \\
\hline 1 & 0.893 & 0.0137 & 0.0091 \\
2 & 0.894 & 0.0068 & 0.0024 \\
3 & 0.900 & 0.0057 & 0.0015 \\
4 & 0.891 & 0.0052 & 0.0012 \\
5 & 0.899 & 0.0050 & 0.0011 \\
6 & 0.899 & 0.0049 & 0.0011 \\
7 & 0.911 & 0.0048 & 0.0010 \\
8 & 0.902 & 0.0047 & 0.0010 \\
\hline
\end{tabular}

Table 2: Coverage, Average and Standard Deviation of Half-Widths for Confidence Intervals Based on $m$ Excursions

\begin{tabular}{|l|l|l|l|}
\hline$m$ & coverage & avg. hw & std. of hw \\
\hline 1 & 0.887 & 0.00447 & 0.001015 \\
3 & 0.883 & 0.00444 & 0.000564 \\
\hline
\end{tabular}

\section{CONCLUSIONS}

We have described a method to construct asymptotically valid confidence intervals for the time-average of a simulated process using a bounded memory of size selected by the user. The method requires (at least) two independent simulations to be run in parallel to center the output in order to avoid storing the entire simulation data. Compared with a straightforward batching scheme, the method appears to have relatively small confidence intervals of low variability for the same memory size (though the batching scheme has excellent coverage properties).

\section{ACKNOWLEDGMENT}

This work was supported in part by the National Science Foundation under grant DMI-9900117.

\section{REFERENCES}

Calvin, J. 2004. Using excursions to analyze simulation output. In preparation.

Glynn, P. W. and D. L. Iglehart. 1990. Simulation output analysis using standardized time series. Mathematics of Operations Research 14:1-16.

Goldsman, D. and B. W. Schmeiser. 1997. Computational efficiency of batching methods. In Proceedings of the 1997 Winter Simulation Conference, ed. S. Andradóttir, K. J. Healy, D. H. Withers, and B. L. Nelson, 202-
207. Piscataway, New Jersey: Institute of Electrical and Electronics Engineers.

Meketon, M. S. and B. W. Schmeiser. 1984. Overlapping batch means: something for nothing? In Proceedings of the 1984 Winter Simulation Conference, ed. S. Sheppard, U. Pooch and C. D. Pegden, 227-230. Piscataway, New Jersey: Institute of Electrical and Electronics Engineers.

Revuz, D. and M. Yor. 1994. Continuous Martingales and Brownian Motion. Berlin: Springer Verlag.

Schruben, L. W. 1983. Confidence interval estimation using standardized time series. Operations Research 31:1090-1108.

Vervaat, W. 1979. A relation between Brownian bridge and Brownian excursion. Annals of Probability 7:141-149.

\section{AUTHOR BIOGRAPHY}

JAMES M. CALVIN is an associate professor in the Department of Computer Science at the New Jersey Institute of Technology. He is an associate editor for ACM Transactions on Modeling and Computer Simulation. In addition to simulation output analysis, his research interests include global optimization and probabilistic analysis of algorithms. 